\title{
Metamaterial-inspired Solution to Lackluster On-Chip Antenna Performance
}

\author{
$\underline{\text { Richard W. Ziolkowski }}$ \\ University of Technology Sydney, Global Big Data Technologies Centre, \\ 81 Broadway, Ultimo NSW 2007, Australia \\ Richard.Ziolkowski@uts.edu.au
}

\begin{abstract}
An electric or magnetic dipole antenna located on the interface between a low and high permittivity dielectric faces the problem that the physics tells us that the majority of the power it emits will be radiated into the high dielectric region. This effect is a significant problem for an on-chip antenna associated with systems-on-chip applications such as mobile computing and embedded systems. It is demonstrated that one can use metamaterial-inspired Huygens antennas to overcome this very practical problem.
\end{abstract}

\section{INTRODUCTION}

A dipole antenna radiating in the presence of a material half-space has been the subject of extensive theoretical investigations for over a century, beginning with the famous results of Sommerfeld in 1909 [1]. It was demonstrated in [2] that the field radiated by an infinitesimal electric source located on the interface between air and a dielectric halfspace would be directed primarily into the dielectric region. It was shown in [3] that similar results occur with either infinitesimal electric or magnetic dipole radiators.

The desire to integrate antenna functions directly on silicon chips in mobile, wireless devices has become intense as those systems move to higher frequencies. It has led to the concept of on-chip antennas (OCAs). In particular, numerous systems-on-chip (SoC) applications such as mobile computing, embedded system, 5G wireless device, and internet-of-things (IoT) applications require compact, high performance radiators, particularly on silicon. Unfortunately, when an efficient dipole antenna is used as an OCA, it is transformed into an inefficient radiator because it will radiate primarily into the silicon substrate. This issue has become a bottleneck for SoC systems and their applications [4]-[6]. The need for improved radiation efficiencies has led to a variety of attempts to overcome this problem, for instance, with impedance matched silicon lenses and even with artificial magnetic conductors (AMCs) in the multilayered stack delineating the OCA [7].

The interface problem is treated here analytically in one dimension (1D) to first demonstrate the OCA problem. It is then used to identify a solution to it based on Huygens sources, i.e., a balanced pair of orthogonal electric and magnetic radiators that produce a broadside cardioid directivity pattern. A variety of Huygens dipole antennas (HDAs) have been successfully realized recently [8-10]. They are based on balanced combinations of the metamaterial-inspired electric (Egyptian axe dipole - EAD) and magnetic (capacitively loaded loop - CLL) near field resonant parasitic (NFRP) elements. These NFRP HDAs provide a potential practical solution to the OCA bottleneck.

\section{Electric CurRent SHeEt on the InTERface Between Two SEMI-INFINITE MediA}

The electromagnetic field radiated by a time harmonic, infinite electric current sheet sandwiched between two semi-infinite media follows naturally from the corresponding homogeneous region solution of the 1D Helmholtz equation. With no lack of generality, the boundary between the two semi-infinite half spaces is assumed to be the $z=0$ plane. The current source is taken to be located on this interface. The current sheet is defined simply as

$$
\vec{J}_{s}=J_{0} \delta(z) \hat{x}
$$

This problem geometry is illustrated in Fig. 1.

With straightforward algebra, the fields and their associated average power density $<\vec{S}_{\omega}>=\frac{1}{2} \operatorname{Re}\left\{E_{\omega x} H_{\omega y}^{*}\right\}$ is obtained in each region. The Poynting vectors are: 


$$
\begin{array}{lll}
<\vec{S}_{\omega, e 2}>=+\frac{1}{2} \frac{\eta_{2}\left|\eta_{1}\right|^{2}}{\left|\eta_{1}+\eta_{2}\right|^{2}} & \left|J_{0}\right|^{2} \hat{z} & \mathrm{z}>0 \\
<\vec{S}_{\omega, e 1}>=-\frac{1}{2} \frac{\eta_{1}\left|\eta_{2}\right|^{2}}{\left|\eta_{1}+\eta_{2}\right|^{2}}\left|J_{0}\right|^{2} \hat{z} & \mathrm{z}<0
\end{array}
$$

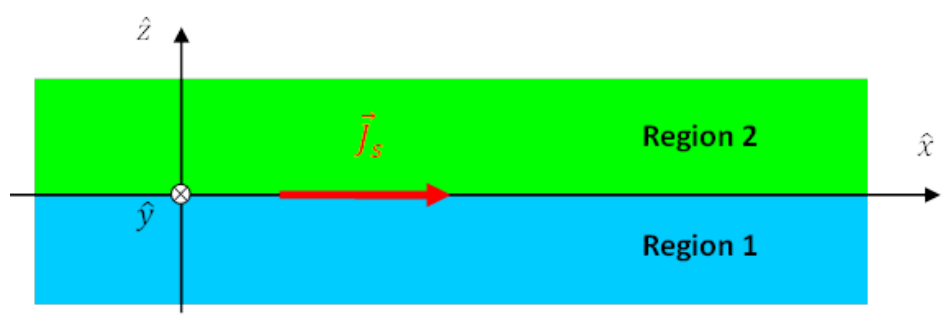

Fig. 1. Infinite electric current sheet configuration

Now consider the case in which both regions are non-magnetic, i.e., dielectrics with $\mu_{2}=\mu_{1}=\mu_{0}$. Let Region 2 be free space, i.e., $\varepsilon_{2}=\varepsilon_{0}$. Let Region 1 be a dielectric with a positive $\varepsilon_{1}=\varepsilon_{r} \varepsilon_{0}$ and, hence, an index of refraction $n_{1}^{2}=\varepsilon_{r 1}$. Consequently, the ratio of the power flows into each region is:

$$
\frac{<\vec{S}_{\omega, e 2}>\cdot \hat{z}}{-<\vec{S}_{\omega, e 1}>\cdot \hat{z}}=\frac{1}{\left|\varepsilon_{r 2}\right|^{3 / 2}}=\frac{1}{\left|n_{2}\right|^{3}}
$$

This $\varepsilon^{3 / 2}$ behavior was established in [2] for an infinitesimal electric dipole antenna and has been identified as a key issue for radiators on air-silicon interfaces [4]-[7]. In particular, silicon has a large relative permittivity, 11.7. Thus, the ratio given by (3) is $1 / 40.02$ which means $97.50 \%(2.50 \%)$ of the radiated power is emitted into the silicon (air). Clearly, a very large portion of the radiated power will then reside in the silicon, Region 1. This behavior significantly impacts the performance characteristics of all OCAs [4]-[7].

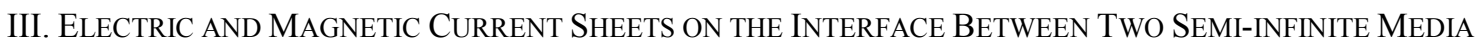

To develop a means to overcome the OCA interface problem, the electromagnetic field radiated when orthogonal infinite electric and magnetic time harmonic current sheets are sandwiched between two semi-infinite media has been considered. This configuration is shown in Fig. 2. It was hoped that a balanced pair of electric and magnetic currents would yield a Huygens field behavior, despite the presence of a radically different permittivity in one of the regions.

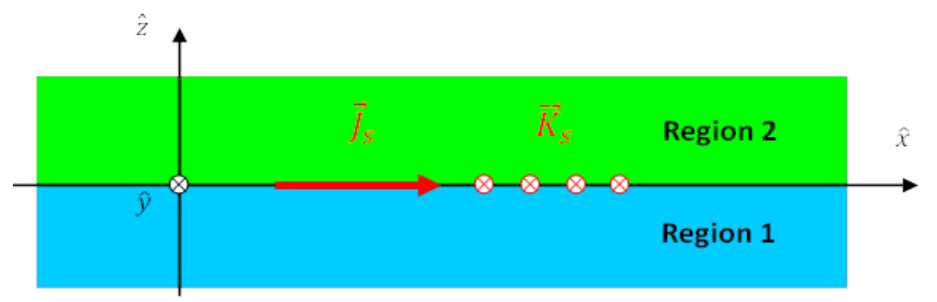

Fig. 2. Configuration in which orthogonal infinite electric and magnetic current sheets lie on the interface between two semi-infinite homogeneous regions

The total fields in both regions follow immediately by the superposition of the two independent electric $\left(\vec{J}_{s}=J_{0} \delta(z) \hat{x}\right)$ and magnetic current sheet $\left(\vec{K}_{s}=K_{0} \delta(z) \hat{y}\right)$ solutions. One finds that if the electric and magnetic currents are properly balanced, i.e., if: $K_{0}=\eta_{2} J_{0}$, the total fields are indeed Huygens fields pointing strictly into Region 2, i.e., 


$$
\begin{aligned}
\vec{E}_{\omega, \text { total } 2}=-\eta_{2} J_{0} e^{-j k_{2} z} \hat{x} & \vec{E}_{\omega, \text { total } 1}=0 \\
\vec{H}_{\omega, \text { total } 2}=-J_{0} e^{-j k_{2} z} \hat{y} & \vec{H}_{\omega, \text { total } 1}=0
\end{aligned}
$$

Consequently, the total average radiated power density in both regions is:

$$
<\vec{S}_{\omega, e 2}>=+\frac{1}{2} \eta_{2}\left|J_{0}\right|^{2} \hat{z} \quad \quad<\vec{S}_{\omega, e 1}>=0
$$

Therefore, with the electric and magnetic current sources balanced with respect to the impedance of Region 2, one recovers the plane wave fields that would exist if both regions were free space, i.e., the power density associated with a plane wave propagating in the $+z$-direction. On the other hand, the balanced condition choice: $K_{0}=-\eta_{1} J_{0}$ has the opposite effect, i.e., zero fields are found in Region 2 and the emitted plane wave is propagating solely into Region 1 .

\section{CONCLUSION}

Actual interface results with ideal and practical metamaterial-inspired HDAs have been obtained and will be described in my presentation. While their finite size cannot match the perfect results of the 1D problem, it can nevertheless be demonstrated that a significant portion of the radiated fields will reside in the upper (air) region. These results indicate that such HDA systems should facilitate more efficient OCA systems that radiate away from the substrate on which they are located. Thus, these HDAs would provide a means to overcome many of the practical design issues and limitations associated with current and future OCAs, as well as impact future onbody IoT wireless sensors and systems. Moreover, these results offer the possibility of not only directing radiated power away from a more complex medium, but also directly into them by properly balancing specific combinations of electric and magnetic current elements.

\section{REFERENCES}

[1] A. Sommerfeld, "Über die ausbreitung der wellen in der drahtlosen telegraphie," Ann. Physik, vol. 333, no. 4, pp. 665736, 1909 (About the propagation of waves in wireless telegraphy

[2] N. Engheta, C. H. Papas, and C. Elachi, "Radiation patterns of interfacial dipole antennas," Radio Sci., vol. 17, no. 6, pp. 1557--1566, Nov.-Dec., 1982.

[3] G. Smith, "Directive properties of antennas for transmission into a material half-space," IEEE Trans. Antennas Propag., vol. 32, no. 3, pp. 232--246, Mar. 1984.

[4] A. Babakhani, X. Guan, A. Komijani, A. Natarajan, and A. Hajimiri, "A 77-GHz phased-array transceiver with on-chip antennas in silicon: Receiver and antennas," IEEE J. Solid-State Circuits, vol. 41, no. 12, pp. 2795--2806, Dec. 2006.

[5] H. M. Cheema and A. Shamim, "The last barrier: On-chip antennas," IEEE Microw. Mag., vol. 14, no. 1, pp. 79--91, Jan.-Feb. 2013.

[6] T. Zwick, F. Boes, B. Göttel, A. Bhutani, and M. Pauli, "Pea-sized mmW transceivers: QFN-based packaging concepts for millimeter-wave transceivers," IEEE Microw. Mag., vol. 18, no. 6, pp. 79—89, Aug. 2017.

[7] X.-Y. Bao, Y.-X. Guo, and Y.-Z. Xiong, "60-GHz AMC-based circularly polarized on-chip antenna using standard 0.18 mm CMOS technology," IEEE Trans. Antennas Propag., vol. 60, no. 5, pp. 2234-2241, May 2012.

[8] R. W. Ziolkowski, "Low profile, broadside radiating, electrically small Huygens source antennas," IEEE Access, vol. 3, pp. 2644-2651, Dec. 2015.

[9] M.-C. Tang, H. Wang, and R. W. Ziolkowski, "Design and testing of simple, electrically small, low-profile, Huygens source antennas with broadside radiation performance," IEEE Trans. Antennas Propag., vol. 64, no. 11, pp. 4607-4617, Nov. 2016.

[10] W. Lin and R. W. Ziolkowski, "Electrically-small, low-profile, Huygens circularly polarized antenna," IEEE Trans. Antennas Propag., vol. 66, no. 2, pp. 636-643, Feb. 2018. 